PROCEEDINGS OF THE

AMERICAN MATHEMATICAL SOCIETY

Volume 128, Number 12, Pages 3465-3469

S 0002-9939(00)05448-4

Article electronically published on June 7, 2000

\title{
ON THE DENSITY OF THE SET OF GENERATORS OF A POLYNOMIAL ALGEBRA
}

\author{
VESSELIN DRENSKY, VLADIMIR SHPILRAIN, AND JIE-TAI YU \\ (Communicated by Wolmer V. Vasconcelos)
}

\begin{abstract}
Let $K[X]=K\left[x_{1}, \ldots, x_{n}\right], n \geq 2$, be the polynomial algebra over a field $K$ of characteristic 0 . We call a polynomial $p \in K[X]$ coordinate (or a generator) if $K[X]=K\left[p, p_{2}, \ldots, p_{n}\right]$ for some polynomials $p_{2}, \ldots, p_{n}$. In this note, we give a simple proof of the following interesting fact: for any polynomial $h$ of the form $\left(x_{i}+q\right)$, where $q$ is a polynomial without constant and linear terms, and for any integer $m \geq 2$, there is a coordinate polynomial $p$ such that the polynomial $(p-h)$ has no monomials of degree $\leq m$. A similar result is valid for coordinate $k$-tuples of polynomials, for any $k<n$. This contrasts sharply with the situation in other algebraic systems.

On the other hand, we establish (in the two-variable case) a result related to a different kind of density. Namely, we show that given a non-coordinate two-variable polynomial, any sufficiently small perturbation of its non-zero coefficients gives another non-coordinate polynomial.
\end{abstract}

\section{INTRODUCTION}

Let $K[X]=K\left[x_{1}, \ldots, x_{n}\right], n \geq 2$, be the polynomial algebra over a field $K$ of characteristic 0 . We denote by $\min \operatorname{deg}(p)$ the minimal degree of non-zero monomials of $p \in K[X]$.

We call automorphic images of $x_{1}$ coordinate polynomials to simplify the language. Similarly, a $k$-tuple of polynomials $\left(p_{1}, \ldots, p_{k}\right), p_{i} \in K[X], k \leq n$, is coordinate if there exists an automorphism of $K[X]$ which sends $x_{1}, \ldots, x_{k}$ respectively to $p_{1}, \ldots, p_{k}$. Equivalently, a $k$-tuple $\left(p_{1}, \ldots, p_{k}\right)$ is coordinate if there are polynomials $p_{k+1}, \ldots, p_{n} \in K[X]$ such that $K\left[p_{1}, \ldots, p_{k}, p_{k+1}, \ldots, p_{n}\right]=K[X]$.

In this note, we give a simple proof of the following interesting fact: the set of coordinate polynomials is dense (in the formal power series topology) in the set of polynomials of the form $\left(x_{i}+q\right), \min \operatorname{deg}(q) \geq 2$. That is, any polynomial of this form can be completed to a coordinate polynomial by monomials of arbitrarily high degree. Actually, our proof yields a somewhat stronger result:

Theorem 1.1. For any $(n-1)$-tuple of polynomials $\left(h_{1}, \ldots, h_{n-1}\right)$ of the form $h_{i}=x_{i}+r_{i}, \operatorname{mindeg}\left(r_{i}\right) \geq 2, i=1, \ldots, n-1$, and any integer $m \geq 2$, there

Received by the editors March 2, 1998 and, in revised form, February 22, 1999.

1991 Mathematics Subject Classification. Primary 13B25; Secondary 16W20.

The first author was partially supported by Grant MM605/96 of the Bulgarian Foundation for Scientific Research.

The third author was partially supported by RGC-Fundable Grant 344/024/0004. 
exists a coordinate $(n-1)$-tuple $\left(p_{1}, \ldots, p_{n-1}\right)$ such that $\operatorname{mindeg}\left(p_{i}-h_{i}\right)>m$, $i=1, \ldots, n-1$.

There is a non-commutative version of Theorem 1.1 (see 44) which involves machinery from representation theory of the general linear group $G L_{n}(K)$. Our proof here is based on simpler ideas and is a consequence of a result of Anick [1].

Theorem 1.1 contrasts sharply with the situation in other, non-commutative, algebras. For example, the set of primitive elements (that is what generators are usually called in a non-commutative setting) of a free Lie algebra of rank 2 is not dense because by a theorem of Cohn [2, all automorphisms of this algebra are linear. Moreover, although the automorphism groups of $K\left[x_{1}, x_{2}\right]$ and $K\left\langle x_{1}, x_{2}\right\rangle$ (the free associative algebra of rank 2) are isomorphic (see e.g. [3]), we have:

Proposition 1.2. The element $u=x_{1}+x_{1} x_{2}$ cannot be completed to a primitive element of $K\left\langle x_{1}, x_{2}\right\rangle$ by monomials of degree higher than 2.

The proof of Proposition 1.2 is based on a characterization of generators of $K\left\langle x_{1}, x_{2}\right\rangle$ as it appears in [6].

Finally, we establish (in the two-variable case) a result related to a different kind of density:

Theorem 1.3. Let $p(x, y)=\sum_{i, j=1}^{m} c_{i j} \cdot x^{i} y^{j}, c_{i j} \in K$, be a non-coordinate polynomial from $K[x, y]$. Let $K=\mathbf{R}$ or $\mathbf{C}$. Then there is an $\varepsilon>0$ such that every polynomial $q(x, y)=\sum_{i, j=1}^{m} c_{i j}^{\prime} \cdot x^{i} y^{j}$ with $\left|c_{i j}-c_{i j}^{\prime}\right|<\varepsilon$ if $c_{i j} \neq 0$ and $c_{i j}^{\prime}=0$ if $c_{i j}=0$, is non-coordinate as well.

\section{Preliminaries}

For background on polynomial automorphisms we refer to the book [3]. Anick [1] proved that, with respect to the formal power series topology, the set $\mathbf{J}$ of endomorphisms of $K[X]$ with an invertible Jacobian matrix is a closed set, and the group of tame automorphisms of $K[X]$ is dense in $\mathbf{J}$. This means that for any polynomial mapping $F=\left(f_{1}, \ldots, f_{n}\right)$ with invertible Jacobian matrix $J(F)$ (i.e. $0 \neq J(F) \in K)$ and for any positive integer $m$, there is a tame automorphism $G=\left(g_{1}, \ldots, g_{n}\right)$ such that the polynomials $f_{i}-g_{i}$ contain no monomials of degree less than $m$. An interpretation of the result of Anick in the language we need is given in [5, Theorem 4.2.7]. We recall some details here briefly.

Let $P_{k}$ be the $K$-vector space of all homogeneous polynomials of degree $k \geq 0$. Let $I_{k}, k \geq 2$, be the semigroup of all polynomial endomorphisms $F=\left(f_{1}, \ldots, f_{n}\right)$ such that $x_{i}$ is the only monomial of $f_{i}$ of degree less than $k ; i=1, \ldots, n$. We write

$$
f_{i}=x_{i}+g_{i}+h_{i},
$$

where $g_{i} \in P_{k}$ is the homogeneous component of $f_{i}$ of degree $k$, and $\operatorname{mindeg}\left(h_{i}\right)>k$.

It turns out that there is a homomorphism $\phi$ of $I_{k}$ onto the direct sum of additive groups $P_{k}^{\oplus n} \cong P_{k} \oplus \ldots \oplus P_{k}$ such that $\phi(F)=\left(g_{1}, \ldots, g_{n}\right)$.

Let $T$ be the group of tame automorphisms of the algebra $K[X]$, and let $\mathbf{S}_{k}$ be the set of all polynomial mappings $S=\left(s_{1}, \ldots, s_{n}\right) \in I_{k}$ such that $s_{i}=x_{i}+g_{i}+h_{i}$, where $g_{i} \in P_{k}$ and $h_{i} \in \sum_{l>k} P_{l}$, with the property

$$
\sum_{i=1}^{n} \frac{\partial g_{i}}{\partial x_{i}}=0
$$


The main step of Anick's proof (see [5], Step 2 of the proof of Theorem 4.2.7) is to show that

$$
\phi\left(T \cap I_{k}\right)=\phi\left(\mathbf{S}_{k}\right)
$$

This yields

Lemma 2.1. For any $S \in \mathbf{S}_{k} \subseteq I_{k}$, there is a tame automorphism $G_{k}$ of $K[X]$ such that $S \circ G_{k}^{-1}$ is in $I_{k+1}$.

\section{Proofs}

Proof of Theorem 1.1. Let $u_{1}, \ldots, u_{n-1}$ be $n-1$ polynomials without constant and linear terms, and let $u_{i j}$ be the homogeneous component of degree $j$ of the polynomial $u_{i} ; j=2, \ldots, m$. Let $k$ be the smallest integer such that $u_{i k} \neq 0$ for some $i$. Let, for example, $i=1$.

Write the partial derivative of $u_{1 k}$ with respect to $x_{1}$ in the form

$$
\frac{\partial u_{1 k}}{\partial x_{1}}=\sum_{j=0}^{k-1} a_{j} x_{n}^{j},
$$

where the polynomials $a_{j}$ do not depend on $x_{n}$. There is a homogeneous polynomial $w_{1 k} \in P_{k}$ such that

$$
\frac{\partial w_{1 k}}{\partial x_{2}}=-\sum_{j=0}^{k-1} a_{j} x_{n}^{j} .
$$

Consider an endomorphism $F_{1 k}$ of the algebra $K[X]$ defined by

$$
F_{1 k}=\left(x_{1}+u_{1 k}, x_{2}, \ldots, x_{n-1}, x_{n}+w_{1 k}\right) .
$$

Clearly, $F_{1 k} \in I_{k}$ and, because of the choice of $w_{1 k}$, also $F_{1 k} \in \mathbf{S}_{k}$. Similarly, we construct endomorphisms

$$
F_{i k}=\left(x_{1}, \ldots, x_{i-1}, x_{i}+u_{i k}, x_{i+1}, \ldots, x_{n-1}, x_{n}+w_{i k}\right)
$$

for some $w_{i k} \in P_{k}, i=2, \ldots, n-1$, such that $F_{i k} \in \mathbf{S}_{k}$. Hence the composition $F_{k}=F_{1, k} \circ \ldots \circ F_{n-1, k}$ also belongs to $\mathbf{S}_{k}$ and by Lemma 2.1 there exists a tame automorphism $G_{k}=\left(g_{1, k}, \ldots, g_{n, k}\right) \in I_{k}$ such that $F_{k+1}=F_{k} \circ G_{k}^{-1} \in I_{k+1}$. Therefore, $g_{i, k}=x_{i}+u_{i, k}+v_{i, k+1}+v_{i}, i=1, \ldots, n-1$, where $v_{i, k+1}$ is homogeneous of degree $k+1$, and $v_{i} \in \sum_{l \geq k+2} P_{l}$.

Continuing this way, we obtain a tame automorphism $G_{k+1} \in I_{k+1}$ such that $g_{i, k+1}=x_{i}+\left(u_{i, k+1}-v_{i, k+1}\right)+w_{i}, i=1, \ldots, n-1$, where $w_{i} \in \sum_{l \geq k+2} P_{l}$.

If we act by the automorphism $G_{k+1}$ on the polynomial $u_{i k}$, we get a polynomial of the form $u_{i k}+s_{i}$, where $s_{i}$ has no homogeneous components of degree less than $(k-1)+(k+1)=2 k>k+1$. Therefore, the automorphism $G_{k+1} \circ G_{k}$ takes $x_{i}$ to $x_{i}+u_{i k}+u_{i, k+1}+$ (terms of higher degree), $i=1, \ldots, n-1$.

In a finite number of steps, we obtain a tame automorphism $G=\left(g_{1}, \ldots, g_{n}\right)$ such that

$$
g_{i}=x_{i}+u_{i}+(\text { terms of higher degree }), i=1, \ldots, n-1 .
$$

This completes the proof of Theorem 1.1 
Proof of Proposition 1.2. By way of contradiction, suppose there is an element $w$ without monomials of degree lower than 3 , such that $u=x_{1}+x_{1} x_{2}+w$ is a primitive element of $K\left\langle x_{1}, x_{2}\right\rangle$.

By Corollary 1.5 of [6], every primitive element of $K\left\langle x_{1}, x_{2}\right\rangle$ is palindromic, i.e., is invariant under the operator $\leftarrow$ that re-writes every monomial backwards. For example, $\left(x_{1} x_{2}\right)^{\leftarrow}=x_{2} x_{1} ;\left(x_{1} x_{2} x_{1} x_{2}^{2}\right)^{\leftarrow}=x_{2}^{2} x_{1} x_{2} x_{1}$, etc.

It is clear that if an element of $K\left\langle x_{1}, x_{2}\right\rangle$ is palindromic, then its every homogeneous component is palindromic, too. Since the homogeneous component of degree 2 of our element $u$ is not palindromic, this yields a contradiction.

Remark. Clearly, the statement of Proposition 1.2 holds for any element $u=x_{1}+$ $a \cdot x_{1} x_{2}+b \cdot x_{2} x_{1} \in K\left\langle x_{1}, x_{2}\right\rangle$, where $a, b \in K$ and $a \neq b$.

A combination of Theorem 1.1 and Proposition 1.2 calls for an example of a coordinate polynomial $p \in K\left[x_{1}, x_{2}\right]$ of the form $x_{1}+x_{1} x_{2}+$ (terms of higher degree); an example like that is given below.

Example. The polynomial

$$
p=x_{1}+x_{1} x_{2}+\frac{1}{4}\left(x_{1} x_{2}^{2}-x_{1}^{2} x_{2}-x_{1}^{3}+x_{2}^{3}\right)-\frac{1}{16}\left(x_{1}+x_{2}\right)^{4}
$$

is coordinate since it is the image of $x_{1}$ under the automorphism $\alpha \beta \alpha^{-2} \beta \alpha$, where $\alpha$ takes $x_{1}$ to $\left(x_{1}+x_{2}\right)$ and fixes $x_{2}$, and $\beta$ fixes $x_{1}$ and takes $x_{2}$ to $\left(x_{2}-\frac{x_{1}^{2}}{4}\right)$.

Proof of Theorem 1.3. Here we use a characterization of two-variable polynomial automorphisms given in [3, Theorem 6.8.5], which implies, in particular, that if $p(x, y) \in K[x, y]$ is a (non-linear) coordinate polynomial, then there is an elementary automorphism of the form $\left\{x \rightarrow x+\lambda \cdot y^{m} ; y \rightarrow y\right\}$ or $\{x \rightarrow x ; y \rightarrow y+$ $\left.\lambda \cdot x^{m}\right\}, \lambda \in K^{*}$, that decreases the degree of $p(x, y)$.

Let $p(x, y)=\sum c_{i j} \cdot x^{i} y^{j}, c_{i j} \in K^{*}$, be a non-linear polynomial. Then the condition in the previous paragraph translates into a system of homogeneous polynomial equations, where $c_{i j}$ are considered indeterminates, and (polynomial) functions of $\lambda$ are considered coefficients (every equation in this system expresses the condition on the coefficient at a particular monomial to be equal to zero). Thus, the set of solutions of this system is a subset of $K^{s}$, where $s$ is the number of non-zero coefficients of our polynomial $p(x, y)$.

If $p(x, y)$ is a coordinate polynomial, then this system has non-zero solutions. If it is not, then the system might or might not have non-zero solutions. If it does not, then, since the set of non-solutions of a polynomial system is an open set (because it is the complement of a closed set), the result follows. Note that the presence of "unfit" solutions (where some of $c_{i j}$ are equal to zero) does not change the openness of the set of non-solutions since it is equivalent to adding to this set several sets of a smaller dimension, and those are always closed sets.

If our system has non-zero solutions, then we apply an elementary automorphism to the polynomial $p(x, y)$ and reduce its degree. The new polynomial is still noncoordinate, and its coefficients are polynomial functions of $c_{i j}$ and $\lambda$. Now applying the same argument to this new polynomial yields the result because continuing the reduction of the degree, we eventually obtain a system without non-zero solutions. 


\section{REFERENCES}

[1] D. J. Anick, Limits of tame automorphisms in $k\left[x_{1}, \ldots, x_{N}\right]$, J. Algebra 82 (1983), 459468. MR 85d:13005

[2] P. M. Cohn, Subalgebras of free associative algebras, Proc. London Math. Soc. (3) 14 (1968), 618-632. MR 29:4777

[3] P. M. Cohn, Free rings and their relations, Academic Press, 1985. MR 87e:16006

[4] V. Drensky, Tame primitivity for free nilpotent algebras, C.R. Math. Rep. Acad. Sci. Canada (Math. Reprorts of the Acad. of Sci.) 14 (1992), 19-24. MR 93b:16045

[5] V. Drensky, Endomorphisms and automorphisms of relatively free algebras, Suppl. ai Rend. Circ. Mat. Palermo 31 (1993), 97-132. MR 94j:16063

[6] V. Shpilrain and J.-T. Yu, On generators of polynomial algebras in two commuting or non-commuting variables, J. Pure Appl. Algebra 132 (1998), 309-315. CMP 99:01

Institute of Mathematics and Informatics, Bulgarian Academy of Sciences, Akad. G. Bonchev Str., Block 8, 1113 Sofia, Bulgaria

E-mail address: drensky@banmatpc.math.acad.bg

Department of Mathematics, University of Hong Kong, Pokfulam Road, Hong Kong E-mail address: shpil@hkusua.hku.hk

Current address: Department of Mathematics, The City College, City University of New York, New York, New York 10027

E-mail address: shpil@groups.sci.ccny.cuny.edu

Department of Mathematics, University of Hong Kong, Pokfulam Road, Hong Kong

E-mail address: yujt@hkusua.hku.hk 